\section{Safety in the laboratory}

SIR-We wish to report briefly on two matters of laboratory safety that have been raised recently in Scientific Correspondence.

The first concerns the claim by Arnold et al. ${ }^{1}$ that the existence of channels and pores in latex gloves revealed by scanning electron microscopy raises doubts about whether such gloves are effective barriers to viruses. It was subsequently pointed out that these channels may be artefacts of sample preparation ${ }^{2}$ and that there is no evidence that viruses are able to pass through latex gloves ${ }^{3.4}$. We have now found that gloves worn in clinical and research laboratories can be readily penetrated by a bacterial virus bacteriophage lambda.

We compared the ability of gloves made of thin polyethylene (PE), polyvinyl chloride (PVC) and latex to be penetrated by this virus both before and after exposure to the disinfectant ethanol (70\%). Sections of the gloves that cover the palm were tested under static conditions. Gloves of PE and PVC failed to be effective barriers to the virus in $40 \%$ and $22 \%$ of trials, respectively, rising to $94 \%$ and $56 \%$ after exposure to alcohol. The trials using latex gloves had a failure rate of $1-2 \%$. Although this study did not involve specific human pathogens, it serves as a warning to those selecting gloves for handling biohazardous materials and illustrates the need for better glove testing standards and quality control.

Our second report is in response to the findings ${ }^{5.6}$ that many solutions of ${ }^{35} \mathrm{~S}$-methionine release volatile radioactive component(s), which can present containment problems. We have not found incubators used for ${ }^{35} \mathrm{~S}$-labelled compounds to be as highly contaminated as those reported ${ }^{5}$. We studied the volatility of ${ }^{35} \mathrm{~S}$-methionine under controlled conditions in open and closed containers. After incubation for 4 hours in closed containers, only $0.01-0.1 \%$ of the ${ }^{35} \mathrm{~S}$ was in volatile form. Although the volatile component was readily soluble in water, its loss - a function of the volume incubated and the vapour pressure — was independent of the evaporation of water from the incubation medium. Reliance on charcoal filters to trap the volatile ${ }^{35} \mathrm{~S}$ compound is unwarranted as binding of the compound to such filters was found to be non-quantitative and reversible.

The release of volatile ${ }^{35} \mathrm{~S}$-component(s) can be controlled by incubating culture vesscls in a sufficient volume of media $\left(14 \mathrm{ml} \mathrm{mCi}^{-1}\right)$ in an incubator with high humidity to reduce the evaporation rate of the incubation media, or by using a $\mathrm{CO}$ - gassed and sealed flask, or by using Petri dishes in sealed plastic bags. Tricine $(50 \mathrm{mM})$, an effective stabilizer, should be added to the media if it is not toxic to the biological agents in culture.

More detailed reports of our findings have been submitted for publication elsewhere.

ROBERT C. KLEIN

ESMERALDA PARTY

The Rockefeller University,

EDWARD L. GERSHEY

1230 York Avenue,

New York, New York 10021, USA

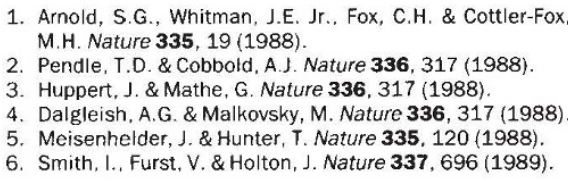

\section{Periodical cicadas}

SIR-Martin and Simon ${ }^{1}$ suggest that 13year cicadas in a large region of central North America are descendants of 17-year cicadas that switched their life-cycle length to 13 years. Their proposal that brood XIX in Illinois, Iowa and Missouri represents a permanent four-year acceleration from brood $\mathrm{X}$ that took place in 1868 is not supported by the historical record.

As Martin and Simon' point out, fouryear accelerations are common in periodical cicada life cycles. But one-year decelerations are also known ${ }^{2}$. I have analysed over 1,500 historical records of periodical cicada emergences dating back to 1804 in Illinois, Indiana, Kentucky and Ohio for patterns of emergence in a given locality. The results show that over 98 per cent of the records are for known broods, four-year accelerations or one-year decelarations from established broods. Moreover, in May/June 1988, two counties in southwestern Ohio and two counties in northern Kentucky witnessed an unexpected emergence of periodical cicadas. The emergence was as heavy as brood $\mathrm{X}$ which emerged in the region a year earlier and involved all three species. Examination of the hatch rate indicated it was as high as brood $\mathrm{X}$, a long-established brood, possibly fore-telling of a future brood which would be numbered brood XI. The brood was heaviest in an area where brood XIV is the predominant brood. Moreover, brood XIV also experienced a one-year deceleration in 1975 in the same area which produced the 1988 emergence. If the unexpected 1988 periodical cicadas were derived from brood XIV it would suggest that the 1988 emergence was a four-year acceleration from the 1975 one-year deceleration. This combination of a four-year acceleration with a one-year deceleration would explain areas, such as northeastern Ohio, where adjacent broods V and VIII occur with three-year differences in their emergences.

Historical records will not indicate mechanism, but they can give important evidence for different models. Lloyd and coworkers $^{3}$ have proposed a mendelian model for four-year accelerations involving a hybridization between 17- and 13-year cicadas. But the Ohio history shows fouryear accelerations in regions where only 17-year cicadas occur. Twelve counties in Illinois and much of southwestern Missouri recorded periodical cicada emergences in 1829,1842 and 1855 indicating that the 13-year life cycle of brood XIX in Illinois and Missouri was established before 1868 (ref. 4). This does not speak against the proposed mechanism presented by Martin and Simon, rather it suggests that the acceleration that gave rise to brood XIX may have occurred earlier than they thought.

Department of Biology,

GENE KRITSKY College of Mount Saint Joseph Mount Saint Joseph,

Ohio 45051, USA

SimON AND MaRTIN REPLY-The complex patterns of periodical cicada emergences are indeed mind-boggling as Kritsky points out. His painstaking historical analyses have shown that despite their much touted reputation, periodical cicadas are not as regular as clockwork. Nevertheless, the irregular emergences are far from random, being either one or four years out of phase. Furthermore, Kritsky's historical data base has proved invaluable in interpreting genetic patterns ${ }^{1,3.5}$. For example, as he notes, hybridization between 13- and 17-year cicadas is not, as proposed $^{3}$, a necessary condition for an acceleration in development.

$\mathrm{We}^{1}$ have presented data which suggest that, in the midwest, a region that had been occupied by both 13- and 17-year cicadas is presently inhabited exclusively by cicadas possessing a 13 -year life cycle but a 17-year genome. These cicadas are genetically identical to 17 -year brood X which disappeared from the area in the first half of this century. Our conclusion is that midwestern 13-year cicadas of brood XIX originated from 17 -year brood $\mathrm{X}$ by a four-year acceleration in development and joined an existing 13-year brood XIX which abutted it to the south.

We agree with Kritsky that the proposed four-year acceleration may not have occurred in 1868 but the presence of 13-year cicadas in the area before 1868 does not rule out this hypothesis. Indeed, a four-year acceleration of midwestern 17-year cicadas to produce 13-year cicadas could have occurred more than once. Despite the particular pathway that produced the geographical and temporal pattern we observed, both Kritsky's historical data and our genetic data indi- 